\title{
Degradable Magnetic Composites for Minimally Invasive Interventions: Device Fabrication, Targeted Drug Delivery, and Cytotoxicity Tests
}

\author{
Journal Article \\ Author(s): \\ Peters, Christian; Hoop, Marcus; Pané, Salvador; Nelson, Bradley J.; Hierold, Christofer \\ Publication date: \\ 2016-01-20 \\ Permanent link: \\ https://doi.org/10.3929/ethz-b-000106798
}

Rights / license:

In Copyright - Non-Commercial Use Permitted

Originally published in:

Advanced Materials 28(3), https://doi.org/10.1002/adma.201503112

Funding acknowledgement:

336456 - Magnetoelectric chemonanorobotics for chemical and biomedical applications (EC) 
Title: Degradable Magnetic Composites for Minimally Invasive Interventions: Device Fabrication, Targeted Drug Delivery, and Cytotoxicity Tests

Author(s), and Corresponding Author(s)*Christian Peters*, Marcus Hoop, Salvador Pané*, Bradley J. Nelson, Christofer Hierold

Christian Peters, Prof. Christofer Hierold

Micro and Nanosystems, ETH Zurich, Tannenstrasse 3, 8092 Zurich, Switzerland

E-Mail: chpeters@ethz.ch, christofer.hierold@micro.mavt.ethz.ch

Marcus Hoop, Dr. Salvador Pané, Prof. Bradley J. Nelson

Institute of Robotics and Intelligent Systems, ETH Zurich, Tannenstrasse 3, 8092 Zurich,

Switzerland

E-Mail: mhoop@student.ethz.ch,vidalp@ethz.ch, bradley.nelson@iris.mavt.ethz.ch

Keywords: degradable composite, superparamagnetic particles, swimming microrobot, targeted drug delivery, cytotoxicity 
Untethered micro and nanoagents are envisioned to travel through the human body and carry out minimally invasive surgeries, deliver drugs, and monitor physiological conditions. While the largest portion of research focuses on the development of novel devices and applications, essential questions regarding localization and recovery of these devices after their use inside the human body remain largely overlooked. In this work, we propose degradable, superparamagnetic hydrogel composites as a one-step solution for device actuation, application, and non-invasive device recovery. The combination of magnetic nanoparticles and a functional, degradable polymer matrix is expected to enable a new generation of magnetic microdevices for a variety of minimally invasive applications. To validate our proposal, a bio-inspired helical microrobot platform mimicking E. coli bacteria is fabricated and actuated using weak rotating magnetic fields. Locomotion based on cork-screw propulsion, targeted drug delivery, and low cytotoxicity of the composite's degradation products are demonstrated.

Robotic devices have the potential to revolutionize therapeutic and diagnostic procedures by reducing invasiveness and enabling medical procedures that would not be possible otherwise. ${ }^{[1]}$ The evolution of robotic tools for biomedical purposes is towards the realization of minimally invasive procedures carried out by untethered micro and nanoagents. Due to unfavorable scaling laws for energy storage, these devices must be powered or actuated externally. ${ }^{[2]}$ Magnetic fields are ideal candidates for this purpose as (1) untethered magnetic devices can be operated in any magnetically transparent environment (vacuum, gaseous environments, as well as conductive and non-conductive, Newtonian and non-Newtonian liquids) and (2) interaction with biological tissue is not problematic. ${ }^{[3,4]}$ A variety of remotely actuatable microdevices for locomotion in liquid environments has been presented. ${ }^{[5,6]}$ One of the most efficient magnetic microrobots that is capable of performing locomotion in liquid environments is the bio-inspired Artificial Bacterial Flagella $(\mathrm{ABF}) \cdot{ }^{[7,8]} \mathrm{ABFs}$ are swimming 
microrobots that adopt the helical tail of the E. coli bacteria and mimic the well-known corkscrew propulsion at the low Reynolds regime. ${ }^{[9]}$ A variety of swimming microrobots has evolved based on this principle and applications such as contact- and non-contact manipulation, cargo transportation, remote sensing, and near IR-tracking have been demonstrated..$^{[3,6,7,10]}$

During future in vivo applications, these devices can be recognized by the immune system, triggering a cascade of specific and non-specific host responses including protein adsorption and platelet activation. ${ }^{[11]}$ While the first may clog the device and impede its functionality, the latter may introduce the life-threatening risk of thrombosis. To prevent these and other nonspecific host responses, the device should be shielded by stealth coatings. ${ }^{[11]}$ Among these coatings, poly (ethylene glycol) (PEG) is considered "the gold standard for stealth polymers". ${ }^{[12]}$ Although these hemocompatible coatings increase blood-circulation time, this protection will be limited, ${ }^{[12]}$ and device recovery must be considered. A lack of efficient device recovery methods is one of the major obstacles that hinders future in vivo application of these devices.

In this work, we suggest a degradable superparamagnetic polymer composite that allows a safe device degradation in vivo and a subsequent degradation product excretion as a noninvasive alternative to manual / surgical device recovery. The superparamagnetic hydrogel composite material can be cross-linked via single and two-photon polymerization (TPP), absorb and release biologically relevant substances, and degrade in aqueous, i.e. physiological, environments. This offers a one-step solution for simple and facile device fabrication, efficient actuation and device application, while rendering post-application device recovery unnecessary. The composite is composed of magnetite $\left(\mathrm{Fe}_{3} \mathrm{O}_{4}\right)$ nanoparticles, high-stealth poly (ethylene glycol) diacrylate (PEG-DA), and pentaerythritol triacrylate (PE-TA). Hydrogel ABFs are fabricated by TPP and wirelessly actuated using weak, rotating magnetic 
fields. Locomotion based on cork-screw propulsion, targeted drug delivery to a model cell line (3T3 fibroblast cells), and low cytotoxicity of the degradation products are demonstrated.

\section{Hydrogel Nanocomposite Copolymers}

Hydrogel nanocomposite (2 \%vol $\mathrm{Fe}_{3} \mathrm{O}_{4}$ ) homopolymer (PEG-DA) composites and copolymer (PEG-DA/PE-TA ratios of 90/10, 75/25, and 50/50 \%vol/\%vol) composites were prepared, drop-casted on transparent glass wafers and loaded into a TPP writing tool as illustrated in Figure 1a. Successful network formation was observed for all copolymers containing at least $25 \%$ vol PE-TA. Three-turn helical structures with helical diameters of 1.5, 2.0, 2.5, and 3.0 $\mu \mathrm{m}$ were fabricated as illustrated in a tilted SEM view in Figure 1b. A typical helical structure with a helical diameter of $3.0 \mu \mathrm{m}$ is illustrated in Figure 1c. The simple and facile fabrication approach allows for the fabrication of large arrays of helical $A B F$ as depicted in Figure 1d. Detailed experimental results are discussed in the Supporting Information 1.

\section{Drug Delivery}

\section{$\underline{\text { Drug release kinetics }}$}

Superparamagnetic hydrogel sheets were crosslinked, immersed in $0.5 \mathrm{mM}$ Methylene Blue (MB) for 24 hours, rinsed with and immersed in phosphate buffer solution (PBS). The drug release kinetics were measured as a fluorescence time series. PBS was frequently exchanged in order to model a perfect sink boundary condition. The drug release kinetics are displayed in Figure 2a for all investigated copolymer ratios.

\section{$\underline{\text { Total amount of absorbed drug(s) }}$}

In order to evaluate the total amount of absorbed MB, the same hydrogel sheets were prepared as denoted above. After rinsing with PBS, hydrogel sheets were immersed in $1 \mathrm{ml}$ of $\mathrm{NaOH}$ 
(1 M) for 24 hours. All polymer sheets fully degraded within this period (The role of $\mathrm{NaOH}$ in the degradation process is discussed below). The MB concentration was evaluated using fluorescence intensity measurements. The obtained signal represents the total drug load and is well in agreement with the results of the drug release kinetics study. For all superparamagnetic hydrogel copolymers, all MB was released from the sheets (see Figure 2b).

\section{$\underline{\text { Swimming tests and targeted drug delivery }}$}

Superparamagnetic hydrogel microrobots were fabricated, immersed in DI water, released from their substrate, and actuated using weak, rotating magnetic fields. The microswimmers were capable of performing locomotion based on cork-screw propulsion (see Supporting Video 1 and Supporting Information 2 for experimental details). For targeted drug delivery experiments, fabricated hydrogel microrobots were immersed in $\mathrm{MB}$ for one hour and subsequently air-dried, released and transferred to a microfluidic test substrate. The test substrate contains two microfluidic pockets fabricated from SU-8 (see Figure 2c). 3T3 fibroblast cells were previously fixated on the substrate and permeabilized. DI-water was added and the swimming microrobots were steered into the microfluidic target pocket. The microrobots remained in the pockets until all water dried out. MB diffuses out of the helical swimmer and starts to stain the surrounding cells. Fluorescence images were taken after the DI water evaporated. The amount of MB that was released from a single device did not result in a sufficient fluorescence signal. Subsequently, a second swimmer was placed inside the microfluidic channel and the experiment was repeated. The accumulated release was sufficient to stain the cells in the target pocket. The cells in the control pocket were not stained. The swimming path as well as the target and control pockets are illustrated in Figure 2c. The target location is again magnified in Figure $2 \mathrm{~g}$. The approach of the second swimmer is illustrated in Figure $2 \mathrm{~d}$ to $\mathrm{f}$. The approach of both swimmers is also illustrated in the 
Supporting Video 1. These results demonstrate, for the first time, targeted drug delivery from a biodegradable swimming microrobot.

\section{Degradation and biocompatibility of degradation products}

All polymer networks discussed in this work are cross-linked acrylate networks containing hydrolytically degradable ester groups (see Figure 3a). Herein, water molecules are able to cleave these ester groups. This results in the formation of poly acrylic acid (PAA) and an alcoholic moiety (PEG or PE), all of which possess a low cytotoxicity and can be metabolized and / or excreted directly from the human body. ${ }^{[13]}$ Upon network degradation, the embedded magnetite is released and can be excreted from the body as well. ${ }^{[14]}$

Accelerated in vitro degradation tests are carried out in the form of sodium hydroxide promoted hydrolysis, also known as saponification. ${ }^{[15]}$ Herein, the ester group is cleaved using aqueous sodium hydroxide, resulting in the formation of sodium poly acrylate (NaPA) instead of PAA as illustrated in Figure 2b.

In order to evaluate the cytocompatibility of the saponificated network, PAA is recovered from NaPA by stoichiometric addition of hydrochloric acid. ${ }^{[16]}$ The degradation products PEG, PE, and PAA are extracted from the aqueous solution by means of phase separation using toluene. The organic phase is dried and white-ish powders are recovered. It has to be noted that the previously embedded nanoparticles remain in the aqueous phase and the cytotoxicity of the entire system cannot be evaluated directly. Instead, saponification and PAA recovery are carried out for pristine, i.e. particle-free, hydrogel pre-polymers only. The corresponding amount of nanoparticles is added to the white-ish powders in form of a ferrofluid (FF, magnetite nanoparticles dissolved in $\gamma$-Butryolactone (GBL)) to match the particle concentration of the fabricated composite devices. The mixture is then dried again and dissolved in PBS. Feed solutions with concentrations between 125 and 2000 ppm degraded superparamagnetic hydrogel copolymer were prepared. The feed solutions were added to 3T3 
fibroblast cells and their viability was measured by MTT assays (see Figure 3c). With 24 hour viabilities above $75 \%$, test results indicate that the degradation products possess a low cytotoxicity. Under a conservative assumption (ABF with $3 \mu \mathrm{m}$ design diameter and $28 \mu \mathrm{m}$ design length approximated by a cylinder with the same feature size), a concentration of 2000 ppm corresponds to about 10 million helical microswimmers per ml, which is an extremely large number. A time sequence of hydrogel microrobots during accelerated degradation in $\mathrm{NaOH}(1 \mathrm{M})$ is illustrated in Figure 3d-j. As excretion pathways for all degradation products exist, ${ }^{[13,14]}$ the discussed superparamagnetic hydrogel copolymer possess all characteristics to qualify as degradable biomaterial with low cytotoxicity and represents an ideal foundation of a magnetic micro or nanoagent.

\section{Discussion and Conclusion}

In this work, we have demonstrated, for the first time, a framework for biodegradable, superparamagnetic hydrogel composites that offers a one-step solution for simple and facile device fabrication, actuation and application, while rendering post-application device recovery unnecessary. To demonstrate the feasibility of our proposal, we fabricated ABFs from fully degradable materials with potential stealth properties, demonstrated targeted drug delivery and in vitro degradation via accelerated hydrolysis. The degradation products possess a low cytotoxicity and potential excretion pathways from the human body. ${ }^{[13,14]}$

As pointed out by Browning et al., the in vivo degradation of hydrolytically degradable materials is also subjected to the macrophage activity, ${ }^{[15]}$ promoting a further examination of the in vivo degradation rate. For practical applications, the polymer material combination may be altered to adjust the degradation time, ${ }^{[17]}$ matching the timeframes of device degradation and device application. Herein, slower degradation may be desirable if the devices support structural integrity of tissue. On the other hand, faster degradation may be advantageous for drug delivery applications. In this context, it should be noted that the loading and delivery 
rates of real drugs are expected to vary from the rates reported for MB. To adjust the drug loading and delivery rates for practical applications, the polymer material may be altered. ${ }^{[18]}$ During drug delivery experiments, we observed that the 3T3 cells around the starting location and along the swimmers' path were stained as well. In agreement with the release kinetics depicted in Figure 2a, this observation indicates that the model drug diffuses out of the devices during the approach of the target pocket. In order to confine the release inside the target pocket only, the implementation of an on-demand release mechanism based on photosensitive nanovalves or hyperthermia may be included in future devices. ${ }^{[19]}$

The simple and facile device fabrication approach presented in this work can be modified to produce different two- and three-dimensional microdevices with arbitrary geometries and may be applied for the fabrication of degradable magnetic sensors and actuators for various applications.

Aside enabling a range of exciting applications, the presented materials and devices provide an alternative solution to post-application device retrieval, promoting degradable superparamagnetic materials as a highly suitable framework for future biomedical applications. In vivo degradation rate, anti-fouling properties, as well as the long-term toxicity must be investigated further before clinical trials can begin.

\section{Experimental Section}

\section{Pre-polymer composition and cross-linking}

PEG-DA 575 and PE-TA were mixed in volume ratios 100 / 0, 90 / 10, 75 / 25, and 50 / $50 \%$ vol / \%vol. FF (particle load $260 \mathrm{mg} / \mathrm{ml} \mathrm{GBL}$, chemicell $\mathrm{GmbH}$ ) was added to achieve a final particle concentration of $2 \%$ vol. Irgacure photoinitiator $(50 \mathrm{mg} / \mathrm{ml}$ polymer $)$ and a photosensitizer $(10 \mathrm{mg} / \mathrm{ml})$ were added. The pre-polymer was mixed and the particles were dispersed in a planetary mixer for 10 minutes. Pristine pre-polymers were prepared in the same manner except that GBL was added instead of the FF. For the fabrication of hydrogel 
$\mathrm{ABFs}$, the pre-polymers were drop-casted onto transparent glass wafers, loaded into a NanoScribe TPP tool, and crosslinked using Perfect Shape Mode 1 with a laser power of 8.6 mW. The explicit writing parameter evaluation is laid out in the Supporting Information. For the fabrication of hydrogel sheets, a modified version of the liquid polymer lithography platform was used with a total exposure dose of $500 \mathrm{~mJ} .^{[20]}$

\section{Drug delivery experiments}

Drug release kinetics: Superparamagnetic hydrogel sheets were dehydrated in vacuum overnight and immersed in $\mathrm{MB}(0.5 \mathrm{mM}$ in DI water) for 12 hours, rinsed with PBS, and immersed in $1 \mathrm{ml}$ PBS. At predefined intervals (5, 10, 20, 30, 60, 120, 240, and 480 minutes), the PBS / MB solution was replaced with fresh PBS, modeling a perfect skink condition.

Total drug absorption: Superparamagnetic hydrogel sheets were dehydrated in vacuum overnight and immersed in $\mathrm{MB}(0.5 \mathrm{mM}$ in DI water) for 12 hours, rinsed with PBS and immersed in $\mathrm{NaOH}(1 \mathrm{M})$ for 24 hours. All polymer sheets were fully degraded at this point.

All fluorescence measurements were repeated at least three times. Details about the fluorescence measurements are given in Supporting Information 3.

Targeted drug delivery: $3 \mathrm{~T} 3$ cells were seeded at a cell density of $10^{6}$ cells and allowed to attach for $24 \mathrm{~h}$ at physiological conditions. Cell media was aspirated and the cells were washed with PBS. Cells were fixed in Formaldehyde (4\%), followed by permeabilization with Triton X-100 (0.1\%). Hydrogel microrobots were incubated for $1 \mathrm{~h}$ in $0.5 \mathrm{mM} \mathrm{MB}$, dried in air, released from their substrate using a Signatone probe station and transferred to the landing zone of the micro channels. DI water was added to the microchannel and the ABF were steered into the target zone using a MagnebotiX NanoMag setup. Rotating magnetic fields with a strength of $15 \mathrm{mT}$ and rotation frequencies between 1 and $5 \mathrm{~Hz}$ were applied. Fluorescence images were taken after $30 \mathrm{~min}$ of incubation using an Olympus IX-81 Fluorescence Microscope with U-MNUA2 and MNBV-2 filter. 


\section{Cytotoxicity of degradation products}

Pristine hydrogel copolymers were crosslinked and immersed in $\mathrm{NaOH}(1 \mathrm{M})$. $\mathrm{HCl}$ was added to recover poly (acrylic acid) from sodium poly acrylate. The solution was ultra-centrifuged to remove impurities. The degraded polymer parts were recovered by phase separation using toluene. The organic phase was vacuum dried for 24 hours and a white-ish powder was recovered. Superparamagnetic FF was added to match the initial particle concentration. The mixture was vacuum-dried to remove GBL and dissolved in PBS. 3T3 fibroblast cells were cultivated in cell culture medium (DMEM, $10 \%$ FCS; $100 \mathrm{x}$ Antimycoticum) at physiological conditions. MTT Cytotoxicity study was conducted in 96 -well plates with $1 \times 10^{4} 3 \mathrm{~T} 3$ cells in culture medium $(100 \mu \mathrm{L})$. Cells were allowed to attach for 4 hours, cell medium was removed, cells were washed with PBS and exposed to the degradation solution. After $24 \mathrm{~h}$ of incubation, the supernatant was replaced by fresh media $(100 \mu \mathrm{L})$ supplemented with MTT $(12 \mathrm{mM})$. After $4 \mathrm{~h}$ of incubation, isopropanol $(100 \mu \mathrm{L})$ and $\mathrm{HCl}(0.04 \mathrm{M})$ were added to the cells. Absorbance measurements were conducted in a microtiter plate reader at $540 \mathrm{~nm}$.

\section{Accelerated degradation tests}

Superparamagnetic hydrogel copolymer ABF were incubated in $\mathrm{NaOH}(1 \mathrm{M})$. Fluorescence images were taken using an Olympus IX-81 Fluorescence Microscope with U-MNUA2 filter.

\section{Supporting Information}

Supporting Information is available from the Wiley Online Library or from the author.

\section{Acknowledgements}

We would like to thank the cleanroom staff of ETH Zurich, Christian Bergemann and Dr. Rainer Quaas (chemicell GmbH) and Dr. Reinhard Schulz (BASF AG) for their support, as 
well as Dr. Selman Sakar for the preparation of the 3T3 fibroblast cells. This work has been funded from the Swiss National Science Foundation (grant nr. 200020-126694).

\section{References}

[1] B. J. Nelson, I. K. Kaliakatsos, J. J. Abbott, Annual Review of Biomedical Engineering 2010, 12, 55.

[2] M. J. Madou, Fundamentals of Microfabrication The Science of Miniaturization, 3rd Edition, Vol. 3, CRC Press, 2011.

[3] K. E. Peyer, L. Zhang, B. J. Nelson, Nanoscale 2013, 5, 1259.

[4] J. Kim, S. E. Chung, S.-E. Choi, H. Lee, J. Kim, S. Kwon, Nat Mater 2011, 10, 747; J. F. Schenck, Progress in Biophysics and Molecular Biology 2005, 87, 185.

[5] L. Zhang, J. J. Abbott, L. Dong, B. E. Kratochvil, D. Bell, B. J. Nelson, Appl. Phys. Lett. 2009, 94; T. Qiu, T.-C. Lee, A. G. Mark, K. I. Morozov, R. Münster, O. Mierka, S. Turek, A. M. Leshansky, P. Fischer, Nat Commun 2014, 5; S. Kim, F. Qiu, S. Kim, A. Ghanbari, C. Moon, L. Zhang, B. J. Nelson, H. Choi, Adv. Mater. 2013, 25, 5863; G. Zhao, M. Pumera, Langmuir 2012, 29, 7411; C. Peters, O. Ergeneman, P. D. W. García, M. Müller, S. Pané, B. J. Nelson, C. Hierold, Adv. Funct. Mater. 2014, 24, 5269; D. Schamel, A. G. Mark, J. G. Gibbs, C. Miksch, K. I. Morozov, A. M. Leshansky, P. Fischer ACS Nano 20148 (9), 8794; A. Ghosh, P. Fischer, Nano Lett. 2009, 9, 2243; P. L. Venugopalan, R. Sai, Y. Chandorkar, B. Basu, S. Shivashankar, A. Ghosh, Nano Lett. 2014, 14, 1968.

[6] O. Ergeneman, G. Dogangil, M. P. Kummer, J. J. Abbott, M. K. Nazeeruddin, B. J. Nelson, Sensors Journal, IEEE 2008, 8, 29.

[7] S. Tottori, L. Zhang, F. Qiu, K. Krawczyk, A. Franco-Obregón, B. J. Nelson, Adv. Mater. 2012, 24, 6, 811.

[8] F. Qiu, L. Zhang, K. E. Peyer, M. Casarosa, A. Franco-Obregon, H. Choi, B. J. Nelson, Journal of Materials Chemistry B 2014, 2, 357; D. J. Bell, S. Leutenegger, K. M.

Hammar, L. X. Dong, B. J. Nelson, "Flagella-like Propulsion for Microrobots Using a Nanocoil and a Rotating Electromagnetic Field", presented at Robotics and Automation, 2007 IEEE International Conference on, 10-14 April 2007, 2007.

[9] H. C. Berg, R. A. Anderson, Nature 1973, 245, 380; E. M. Purcell, American Journal of Physics 1977, 45, 3.

[10] A. Servant, F. Qiu, M. Mazza, K. Kostarelos, B. J. Nelson, Adv. Mater. 2015, 27, 19, 2981.

[11] Z. Yuquan, Y. Kai, F. L. L. Benjamin, E. B. Donald, N. K. Jayachandran, in Handbook of Biofunctional Surfaces, Pan Stanford Publishing, 2013, 923.

[12] K. Knop, R. Hoogenboom, D. Fischer, U. S. Schubert, Angewandte Chemie International Edition 2010, 49, 6288.

[13] K.-F. Mück, O. Christ, H.-M. Kellner, Die Makromolekulare Chemie 1977, 178, 2785; R. Webster, V. Elliott, B. K. Park, D. Walker, M. Hankin, P. Taupin, in PEGylated Protein Drugs: Basic Science and Clinical Applications, (Ed: F. Veronese), Birkhäuser Basel, 2009, 127; I. W. F. Davidson, H. S. Miller, F. J. Dicarlo, J. Pharm. Sci. 1971, 60, 274.

[14] Wahajuddin, S. Arora, International Journal of Nanomedicine 2012, 7, 3445. 
[15] M. B. Browning, E. Cosgriff-Hernandez, Biomacromolecules 2012, 13, 779.

[16] A. Galperin, T. J. Long, B. D. Ratner, Biomacromolecules 2010, 11, 2583.

[17] J. Zhu, Biomaterials 2010, 31, 4639; J. Zhu, R. E. Marchant, Expert Review of Medical Devices 2011, 8, 607.

[18] D. E. Cutright, B. Perez, J. D. Beasley Iii, W. J. Larson, W. R. Posey, Oral Surgery, Oral Medicine, Oral Pathology 1974, 37, 142; T. Niwa, H. Takeuchi, T. Hino, N. Kunou, Y. Kawashima, Journal of Controlled Release 1993, 25, 89.

[19] A. Koçer, M. Walko, W. Meijberg, B. L. Feringa, Science 2005, 309, 755; I. Iscla, C. Eaton, J. Parker, R. Wray, Z. Kovács, P. Blount, Biosensors 2013, 3, 171; C. Brazel, Pharm Res 2009, 26, 644.

[20] C. Peters, S. Fusco, B. J. Nelson, C. Hierold, "Backside Liquid Phase Photolithography for Fabricating Self-Organizing Hydrogel Bilayers", presented at EuroSensors 2012, Krakow, Poland, 2012. 
a)
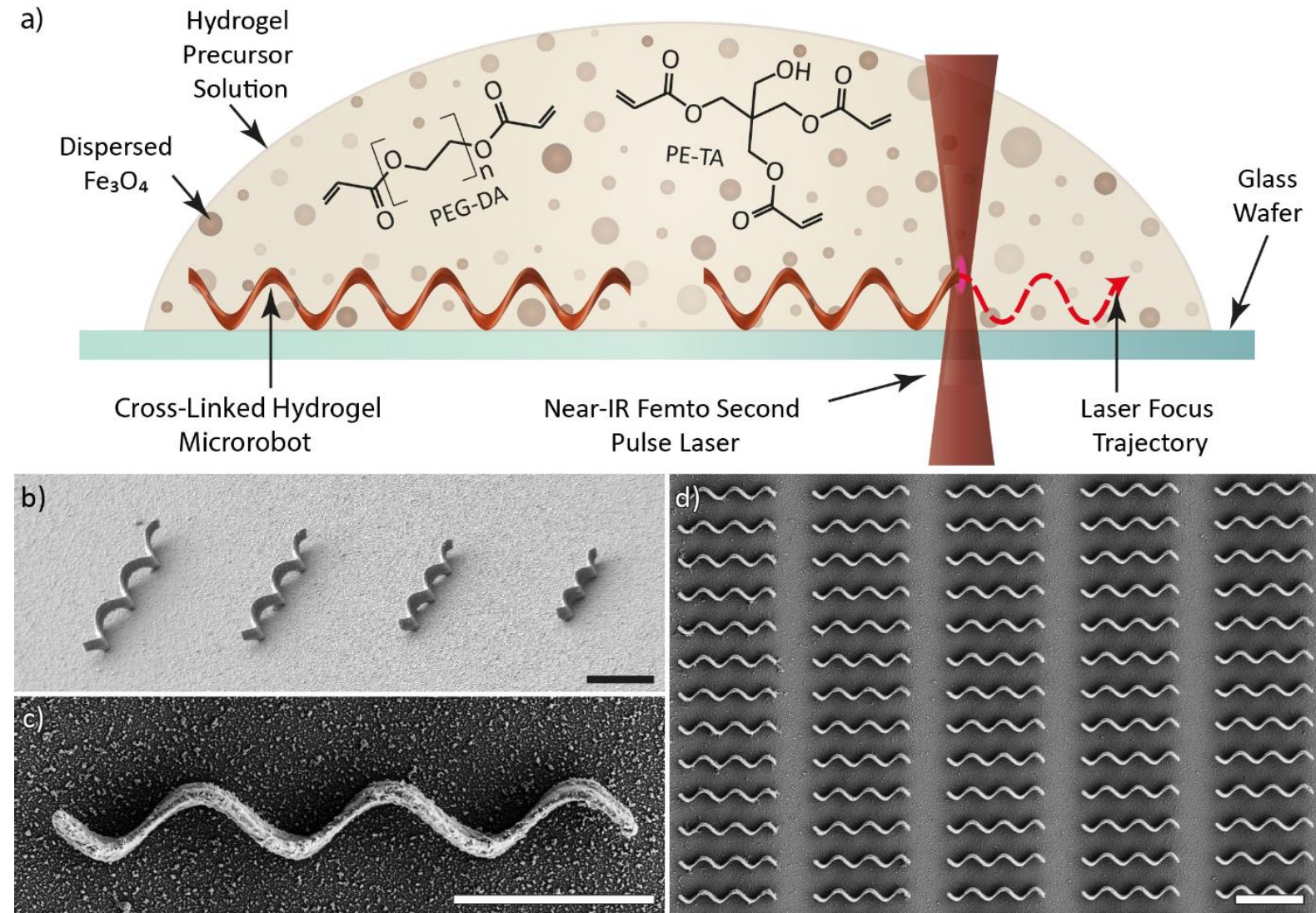

Figure 1 a) Fabrication of superparamagnetic hydrogel microrobots by TPP; b) Helical test patterns with diameters between 1.5 and $3.0 \mu \mathrm{m}$ and lengths between 14 and $28 \mu \mathrm{m}$ in a tilted SEM image; c) SEM Top-view of a helical swimming microrobot; d) SEM top-view of an array of helical swimming microrobots; all displayed micro robots in figures b-d fabricated from a 50/50 \%vol PEG-DA/PE-TA solution, all scale bars are $10 \mu \mathrm{m}$. 

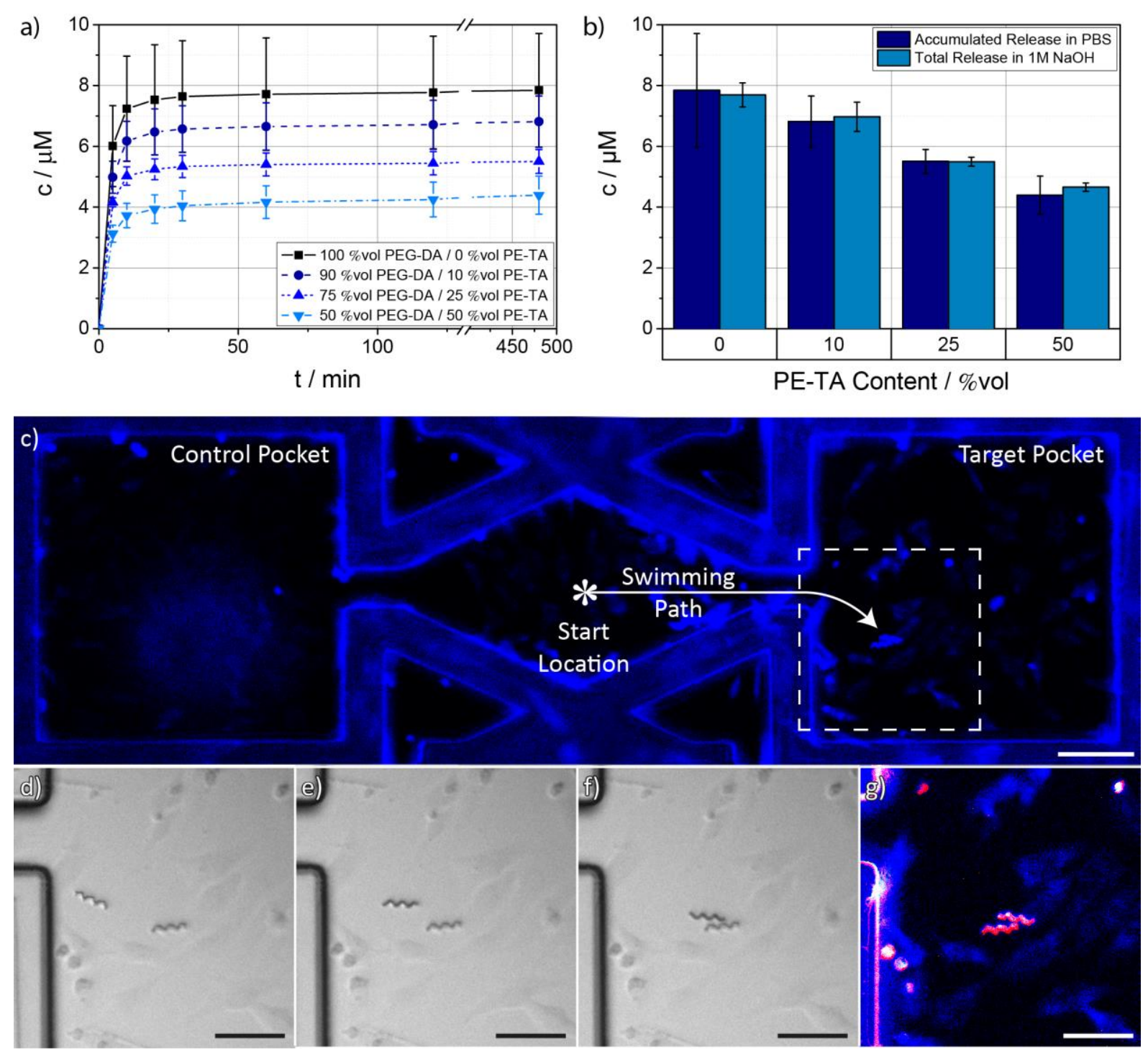

Figure 2 a) MB release kinetics of $9 \times 9 \times 0.03 \mathrm{~mm}$ hydrogel sheets; b) Accumulated total release after $8 \mathrm{~h}$ in PBS vs. total release in $1 \mathrm{M} \mathrm{NaOH}$ after $24 \mathrm{~h}$. c) False color fluorescence image of the microfluidic test field, swimmers are placed in the center and steered into the target pocket, cells in the target pocket are stained by MB, which diffuses out of the helical swimmers, scale bar is $100 \mu \mathrm{m}$; d-f) Approach sequence of swimmer two; g) Dual false color SEM image of the helical swimmers (magenta) and the stained cells around them (blue), Remaining scale bars are $50 \mu \mathrm{m}$. 
a) Ester Hydrolysis

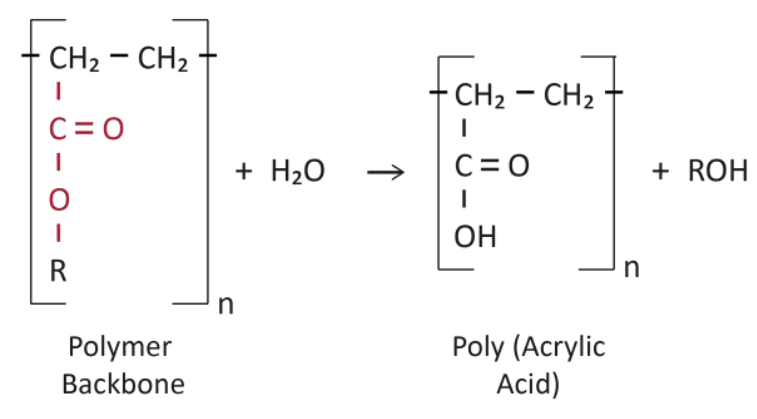

b) Ester Saponification
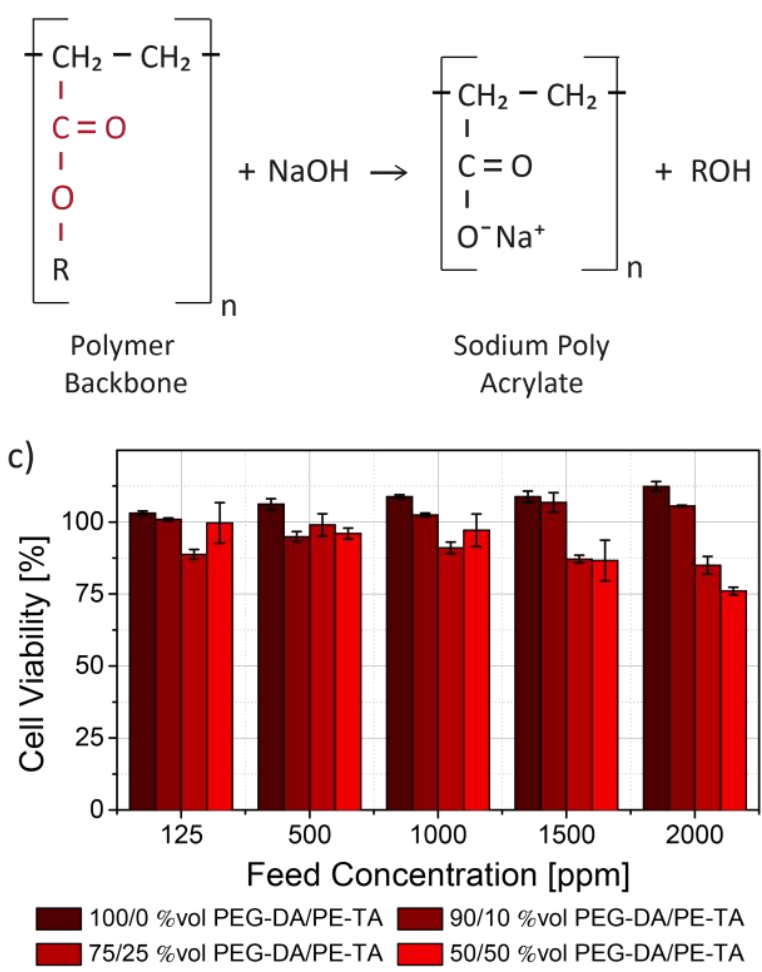
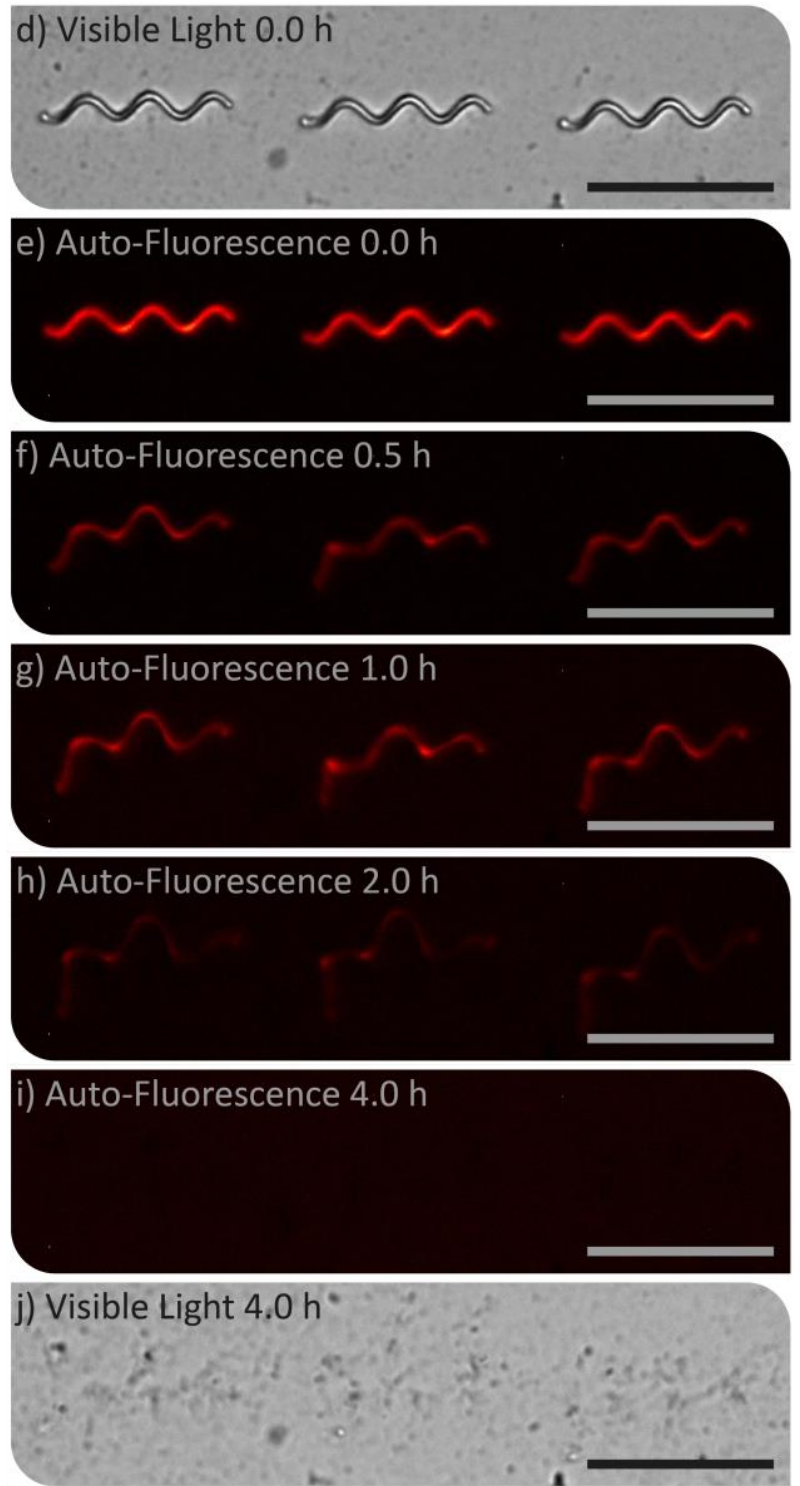

Figure 3 a) Hydrolytic cleavage of ester bonds (red), $\mathrm{ROH}$ = poly (ethylene glycol) or pentaerythritol;

b) Accelerated ester cleavage based on saponification; c) Cell viability after incubation with diluted degradation products; d,j) Light microscopy images before and after saponification; e-i) Autofluorescence saponification time series of superparamagnetic hydrogel swimming microrobots; All scale bars are $25 \mu \mathrm{m}$. 


\section{Supporting Information 1: Fabrication}

Title: Degradable Magnetic Composites for Minimally Invasive Interventions: Device Fabrication, Targeted Drug Delivery, and Cytotoxicity Tests

Author(s), and Corresponding Author(s)* Christian Peters*, Marcus Hoop, Salvador Pané, Bradley J. Nelson, Christofer Hierold

Author(s), and Corresponding Author(s)*Christian Peters*, Marcus Hoop, Salvador Pané,

Bradley J. Nelson, Christofer Hierold

\section{Parameter evaluation for the fabrication of swimming microrobots}

\section{Device design}

Two-photon polymerization (TPP) of superparamagnetic Epon and acrylate-based homo- and co-polymers has been introduced as a suitable approach for the fabrication of non-degradable helical structures. ${ }^{[1]}$ It is also applied for the fabrication of all swimming microrobots discussed in this work. All helical devices were fabricated from a single writing trajectory, i.e. the TPP laser was moved along the helical shape only once. Helical writing trajectories with diameters of $1.5,2.0,2.5$, and $3.0 \mu \mathrm{m}$ were generated using a custom MATLAB code. All helical devices discussed in this work are three-turn helices with a filament pitch of $45^{\circ}$, resulting in device lengths between 14.1 and $28.3 \mu \mathrm{m}$.

\section{Materials and methods}

Poly (ethylene glycol) diacrylate (PEG-DA) 575 and pentaerythritol triacrylate (PE-TA) were purchased from Sigma Aldrich, Switzerland. Superparamagnetic ferrofluid (FF) with a particle content of $260 \mathrm{mg} / \mathrm{ml}$ was obtained from Chemicell GmbH Berlin, Germany. All chemicals were used as received. PEG-DA and PE-TA were mixed in volume ratios 90/10, 
75/25, and 50/50 \%vol/\%vol. FF was added to pristine PEG-DA and to the PEG-DA/PE-TA solutions to achieve a final particle concentration of $2 \%$ vol with respect to the total polymer volume. Irgacure photoinitiator (50 mg per ml polymer solution) and a photosensitizer (10 mg per ml polymer solution) were added. The pre-polymer was mixed and the particles were dispersed in a Hauschild DAC planetary mixer for 10 minutes. Pristine pre-polymers were prepared in the same manner except that no FF was added. These pre-polymers were dropcasted onto transparent glass wafers and loaded into a NanoScribe TPP tool. Cross-linking was carried out in Perfect Shape 1 mode. The laser power was increased from 1.0 to $8.6 \mathrm{~mW}$ in $0.2 \mathrm{~mW}$ steps. A laser power was considered as sufficient if all helical configurations (with diameters between 1.5 and $3.0 \mu \mathrm{m}$ ) were successfully cross-linked at the same time. Fabrication results were accessed using optical microscopy and secondary electron microscopy.

\section{Results}

Helical configurations were fabricated for all pristine polymer solutions, i.e. pristine PEG-DA and pristine PEG-DA-co-PE-TA copolymers. The minimum required laser power decreases from $8.6 \mathrm{~mW}$ (pristine PEG-DA) to $3.0 \mathrm{~mW}$ (PEG-DA-co-PE-TA with a ratio of $50 / 50 \% \mathrm{vol} / \% \mathrm{vol})$. In contrast, superparamagnetic helical devices were only fabricated for copolymers that contained at least $25 \%$ vol PE-TA. Minimal laser powers of $8.6 \mathrm{~mW}$ and 5.0 $\mathrm{mW}$ were observed for volume ratios of 75/25 and 50/50 \%vol/\%vol, respectively. All results are summarized in Figure S1.

All helical structures are three turn devices with a pitch angle of $45^{\circ}$ (see above). For device diameters between 1.5 and $3.0 \mu \mathrm{m}$, this corresponds to arc lengths between 20 and $40 \mu \mathrm{m}$. The Perfect Shape 1 writing mode operates at an average writing speed between 40 and 50 $\mu \mathrm{m} / \mathrm{s}$, implying writing times for a single device in the order of 0.5 to 1.0 second per device. Within one day, between 86,000 and 172,000 devices may be fabricated. 


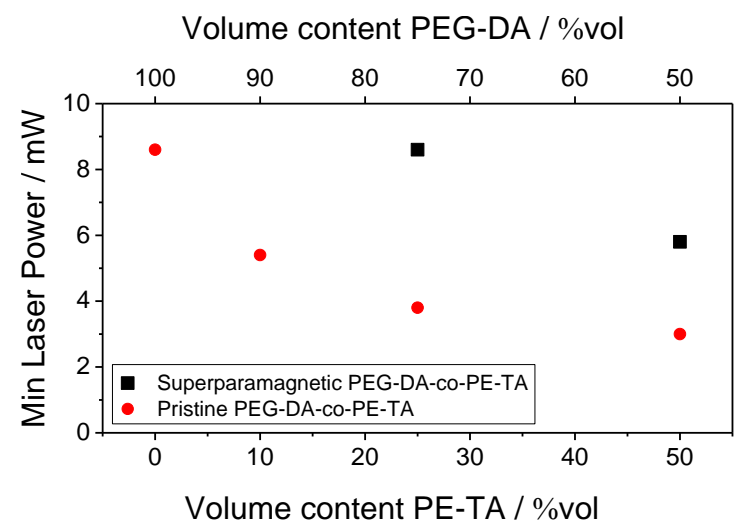

Figure S1 Minimum laser power required to crosslink pristine and superparamagnetic helical swimming microrobots in dependence of the polymer solution composition; Please note that the volume content is always calculated versus the total polymer volume, i.e. excluding particle and solvent volume.

\section{References}

[1] C. Peters, O. Ergeneman, B. J. Nelson, C. Hierold, "Superparamagnetic swimming microrobots with adjusted magnetic anisotropy", presented at Micro Electro Mechanical Systems (MEMS), 2013 IEEE 26th International Conference on, 20-24 Jan. 2013, 2013; C. Peters, O. Ergeneman, P. D. W. García, M. Müller, S. Pané, B. J. Nelson, C. Hierold, Adv. Funct. Mater. 2014, 24, 5269; M. Suter, L. Zhang, E. Siringil, C. Peters, T. Luehmann, O. Ergeneman, K. Peyer, B. Nelson, C. Hierold, Biomed Microdevices 2013, 15, 997; V. C. C. Peters, S. Pané, B.J. Nelson, C. Hierold, "Superparamagnetic Hydrogels of Two-Photon Polymerization and Their Application of the Fabrication of Swimming Microrobots", presented at 18th International Conference on Solid-State Sensors, Actuators and Microsystems Transducers 2015, Anchorage, Alaska, 2015; H. Xia, J. Wang, Y. Tian, Q.-D. Chen, X.-B. Du, Y.-L. Zhang, Y. He, H.-B. Sun, Adv. Mater. 2010, 22, 3204. 


\section{Supporting Information 2: Actuation}

Title: Degradable Magnetic Composites for Minimally Invasive Interventions: Device Fabrication, Targeted Drug Delivery, and Cytotoxicity Tests

Author(s), and Corresponding Author(s)* Christian Peters*, Marcus Hoop, Salvador Pané, Bradley J. Nelson, Christofer Hierold

Author(s), and Corresponding Author(s)*Christian Peters*, Marcus Hoop, Salvador Pané,

Bradley J. Nelson, Christofer Hierold

\section{Characterization of forward velocities}

Setup

Forward velocity characterization was carried out in a custom Helmholtz Manipulator consisting of three orthogonal Helmholtz coil pairs with diameters of 37, 49, and $64 \mathrm{~mm}$, respectively. The coils are placed in the center of a probe station (Signatone S1160). The coils are calibrated to generate a homogeneous magnetic field with a maximum field strength of $10 \mathrm{mT}$. The probe station further contains a water tank in the center of the coil setup. The fabricated devices were immersed in the water tank and removed from their substrate using a tungsten probe mounted on a manual xyz stage (Signatone S926). The devices were transferred within the water tank to a blank silicon die, on which the swimming experiments were carried out. Forward velocities were evaluated using a custom image recognition software.

\section{Results}

The forward velocities of a typical hydrogel robot with a design diameter of $3 \mu \mathrm{m}$ are illustrated in Figure S2 for magnetic field strengths of 5 and $8 \mathrm{mT}$. Maximum forward 
velocities of 5.6 and $10.2 \mu \mathrm{m}$ per second were achieved at step-out frequencies of 3.5 and $7 \mathrm{~Hz}$, respectively (all data points are at least threefold measurement results). Considering a device length of $28.3 \mu \mathrm{m}$, the maximum forward velocities correspond to 0.2 and 0.36 body lengths per second, which is a typical value for superparamagnetic ABF. ${ }^{[1]}$

Using a slender helix approximation (filament diameter $1.0 \mu \mathrm{m}$ ), ${ }^{[2]}$ the attained rotation frequencies can translated into magnetic torques of 8.5 and $17.0 \mathrm{aNm}$.

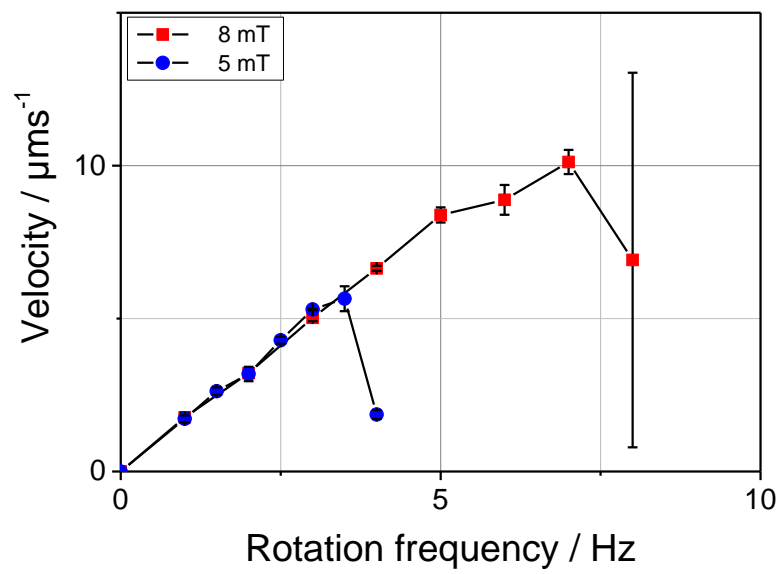

Figure S2 Forward velocity vs. rotation frequency of a typical hydrogel robot (25/75 \%vol/\%vol PE-TA/PEG-DA, diameter $3 \mu \mathrm{m}$, length $28.3 \mu \mathrm{m})$ for rotating magnetic fields of 5 and $8 \mathrm{mT}$.

\section{Discussion}

The demonstrated forward velocities are relatively slow in comparison so other swimming microrobots such as (1) ABFs fabricated by TPP of pristine SU-8 photoresist and subsequent evaporation of magnetic material (10 to 15 body lengths / second), (2) ABFs fabricated by template assisted electrodeposition (3 body lengths / second), or (3) artificial magnetotactic bacteria propelled by catalytic bubble ejection ( 6 to 18 body lengths / second). ${ }^{[3]}$ 
In a previous work, the alignment of the embedded particles has yielded a nearly four-fold increase in forward velocity. ${ }^{[1]}$ In a second, separate work, swimming microrobots with $4 \%$ vol particle load were introduced, ${ }^{[4]}$ in comparison to the $2 \%$ vol particle load employed in this work. Since the magnetic torque is proportional to the magnetization, i.e. magnetic volume, doubling the particle content is expected to double the maximum forward velocity. In turn, a combination of increased particle load and aligned particle configurations may enable forward velocities in the order of 1.6 to 2.9 body lengths per second.

\section{References}

[1] C. Peters, O. Ergeneman, B. J. Nelson, C. Hierold, "Superparamagnetic swimming microrobots with adjusted magnetic anisotropy", presented at Micro Electro Mechanical Systems (MEMS), 2013 IEEE 26th International Conference on, 20-24 Jan. 2013, 2013, 564.

[2] J. J. Abbott, M. Cosentino Lagomarsino, L. Zhang, L. Dong, B. J. Nelson, The International Journal of Robotics Research 2009.

[3] S. Tottori, L. Zhang, F. Qiu, K. Krawczyk, A. Franco-Obregón, B. J. Nelson, Adv. Mater. 2012, 24, 6, 811; M. A. Zeeshan, R. Grisch, E. Pellicer, K. M. Sivaraman, K. E. Peyer, J. Sort, B. Özkale, M. S. Sakar, B. J. Nelson, S. Pané, Small 2014, 10, 7, 1284; G. Zhao, M. Pumera, Langmuir 2012, 29, 7411; G. Zhao, S. Sanchez, O. G. Schmidt, M. Pumera, Chemical Communications 2012, 48, 10090.

[4] M. Suter, L. Zhang, E. Siringil, C. Peters, T. Luehmann, O. Ergeneman, K. Peyer, B. Nelson, C. Hierold, Biomed Microdevices, 2013, 15, 6, 997 


\section{Supporting Information 3: Fluorescence spectra and calibration data}

Title: Degradable Magnetic Composites for Minimally Invasive Interventions: Device Fabrication, Targeted Drug Delivery, and Cytotoxicity Tests

Author(s), and Corresponding Author(s)* Christian Peters*, Marcus Hoop, Salvador Pané, Bradley J. Nelson, Christofer Hierold

Author(s), and Corresponding Author(s)*Christian Peters*, Marcus Hoop, Salvador Pané,

Bradley J. Nelson, Christofer Hierold

\section{Fluorescence spectra and calibration data}

Fluorescence spectra and calibration curves for Methylene Blue (MB) in PBS and $\mathrm{NaOH}$ (1M) are presented below. All solutions were diluted with PBS or $\mathrm{NaOH}$ solution (1 M) from a $\mathrm{MB}$ stock solution $(0.5 \mathrm{mM}$ in DI water) according to the dilution tree presented in Figure S3. Fluorescence spectra and calibration data are presented in Figure S4 and Figure S5.

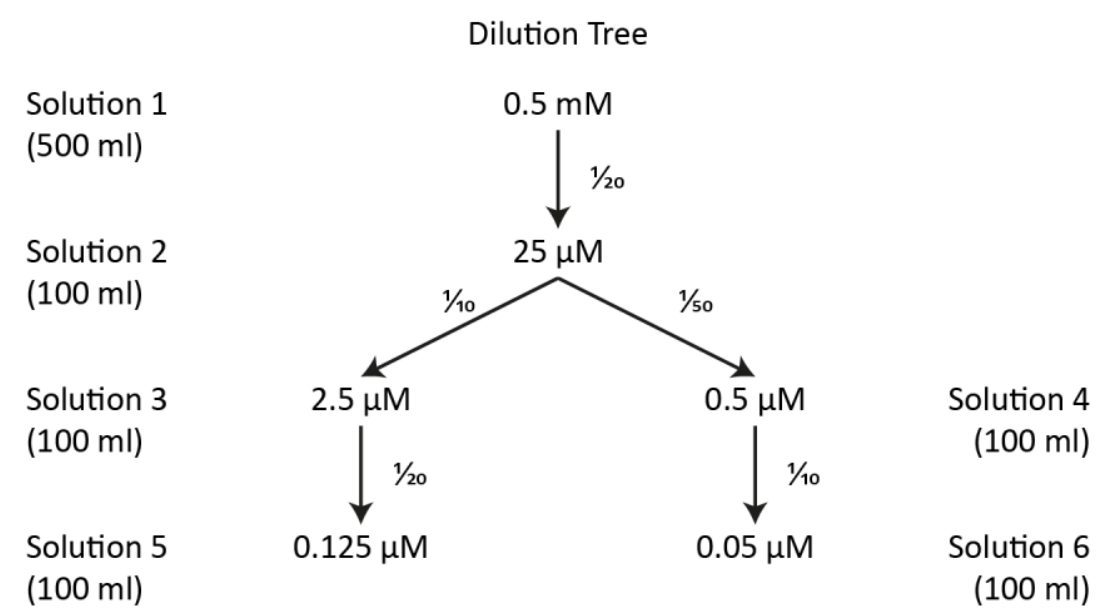

Figure S3 Methylene Blue dilution tree starting from a $0.5 \mathrm{mM}$ stock solution, dilution ratios are illustrated as fractions beside the dilution branches; Dilution tree is applicable for PBS solution as well as $\mathrm{NaOH}(1 \mathrm{M})$ solution. 


\section{$M B$ in PBS}
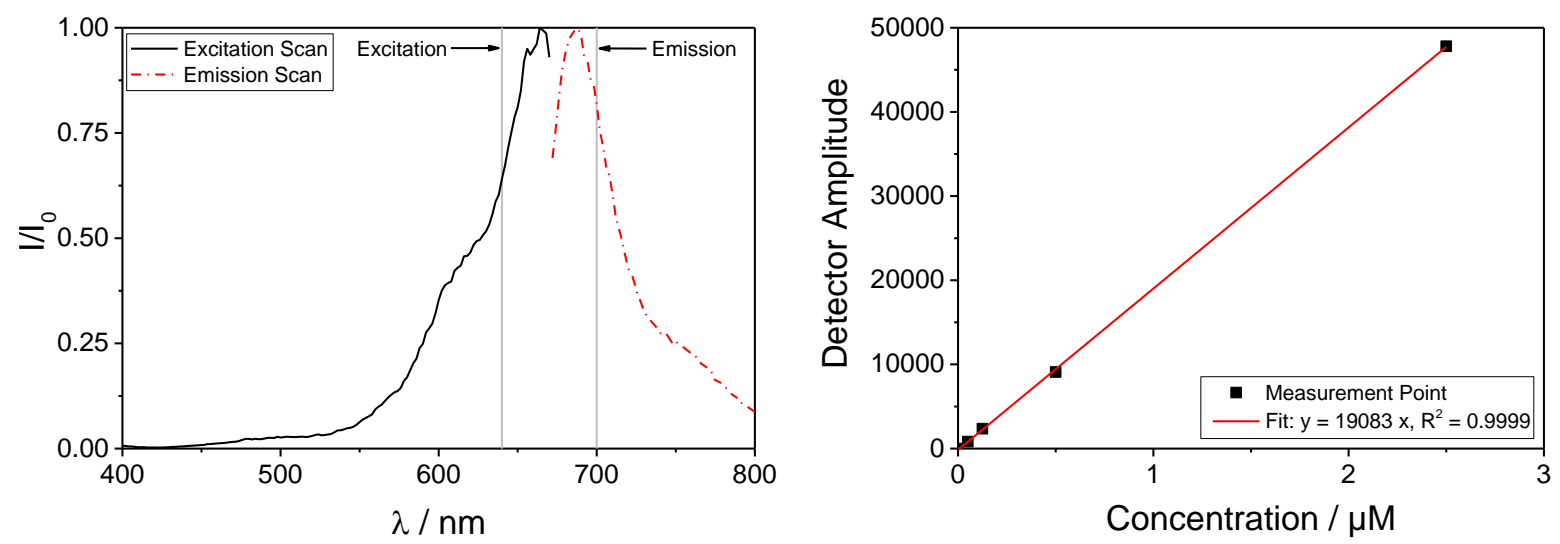

Figure S4 Left: Normalized excitation and emission scans for MB in PBS, Excitation wavelength: 640 nm, Emission wavelength: 700 nm; Right: Calibration curve.

\section{$\mathrm{MB}$ in $\mathrm{NaOH}(\mathbf{1} \mathrm{M})$}
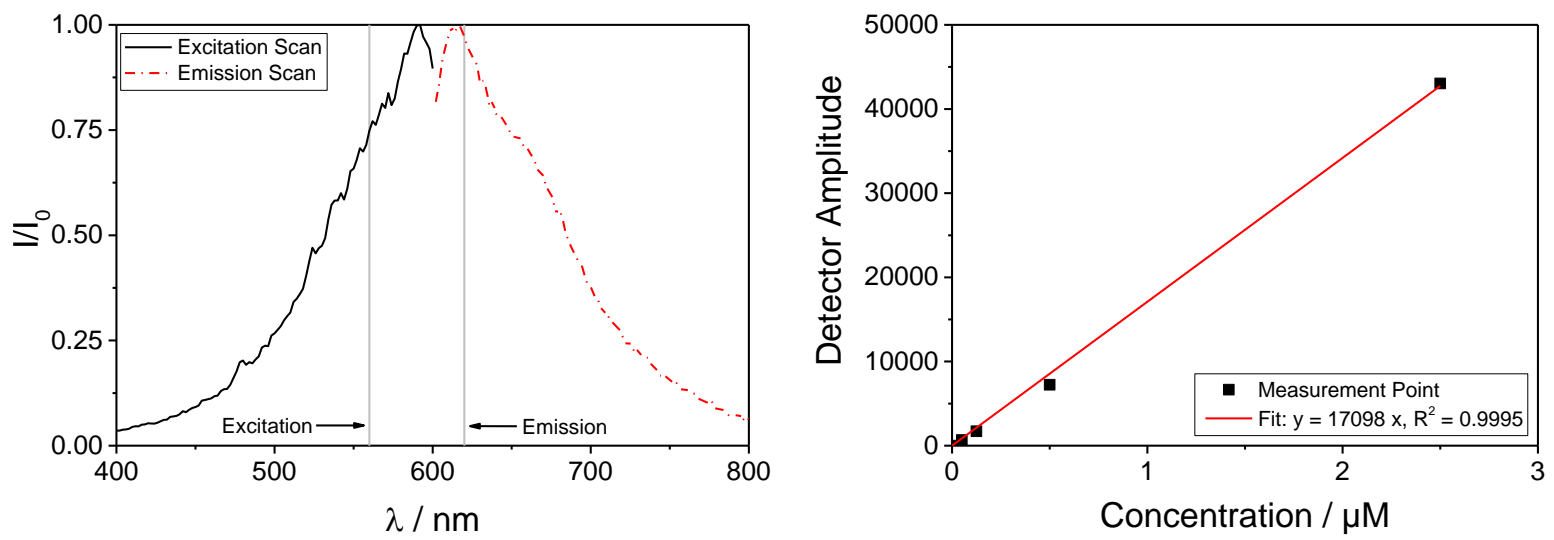

Figure S5 Left: Normalized excitation and emission scans for $\mathrm{MB}$ in $\mathrm{NaOH}$ (1 M), Excitation wavelength: $560 \mathrm{~nm}$, Emission wavelength: $620 \mathrm{~nm}$; Right: Calibration curve. 